\title{
Real-time Ultrafine Aerosol Measurements from Wastewater Treatment Facilities
}

\section{(Supporting Information)}

\author{
P. Piqueras ${ }^{1,2}$, F. Li ${ }^{3}$, V. Castelluccio ${ }^{1,2}$, M. Matsumoto ${ }^{1, \dagger}$ and A. Asa- \\ Awuku ${ }^{1,2, *}$
}

[1] \{Department of Chemical and Environmental Engineering, Bourns College of Engineering, University of California, Riverside, CA 92521, USA\}

[2]\{Bourns College of Engineering, Center for Environmental Research and Technology (CE-CERT), Riverside, CA 92507, USA

[3] School of Environmental Science and Engineering, Nanjing University of Information Science and Technology, 219 Ningliu Road, Nanjing 210044, China\} $[\dagger]\{$ Now at the School of Engineering, University of California, Merced, CA 95343\} Correspondence to: A. Asa-Awuku, 900 University Avenue, Bourns A219, Riverside, CA (akua@engr.ucr.edu; (951) 781-5785)

Content: Two pages and three figures 
To understand the contribution of water to the wet aerosol size, a dry-wet particle comparison experiment was performed by operating two SMPS simultaneously connected to the bioreactor. One line had a copper heating coil attached to it at $50{ }^{\circ} \mathrm{C}$ which dried the particles and the other one did not. The size distribution between the wet and dry particle measurements were minimal (Fig S1).

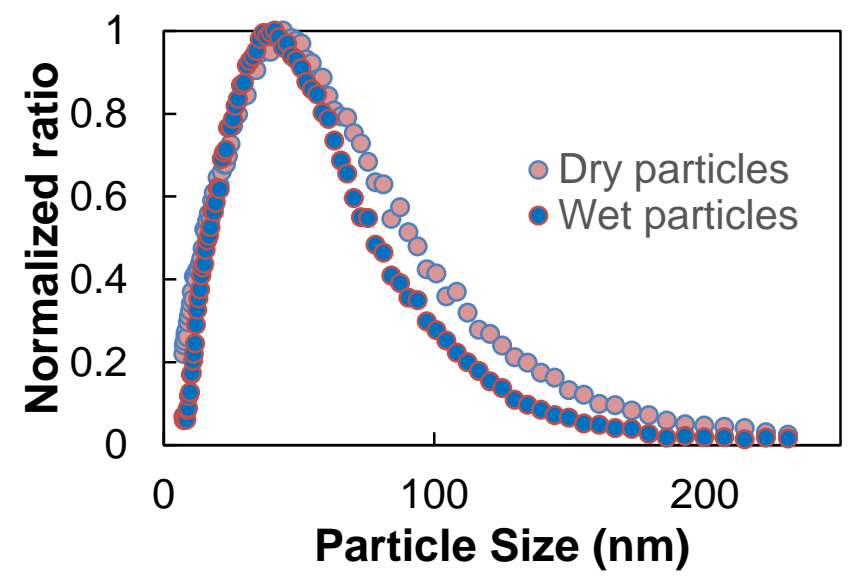

Figure S1: Wet and dry particle concentration measurements taken from the bioreactor.

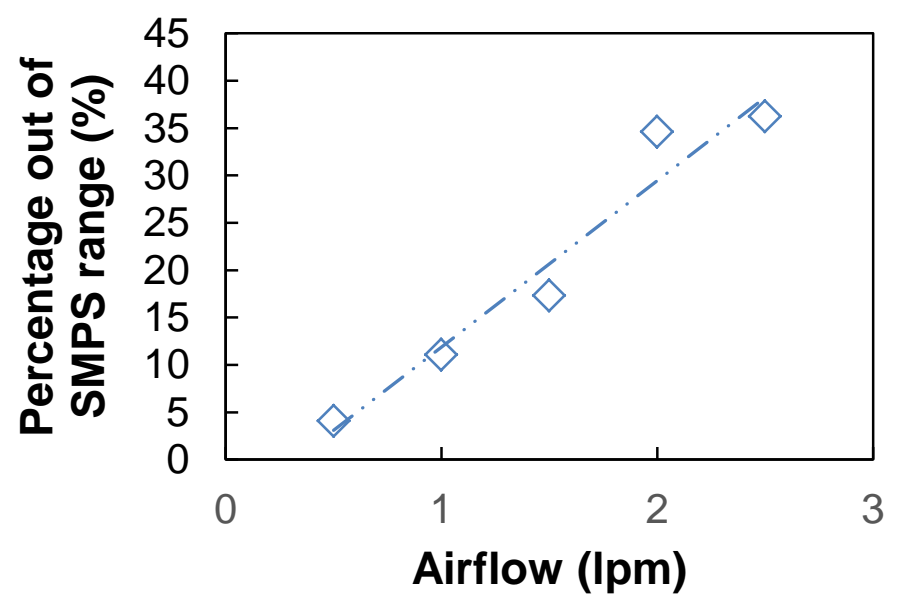


Figure S2: Percentage of particles measured outside of the SMPS range as airflow increased. A significant positive relationship is observed $(r(4)=0.94, p<0.05)$ with a standard error of $5.25 \%$.

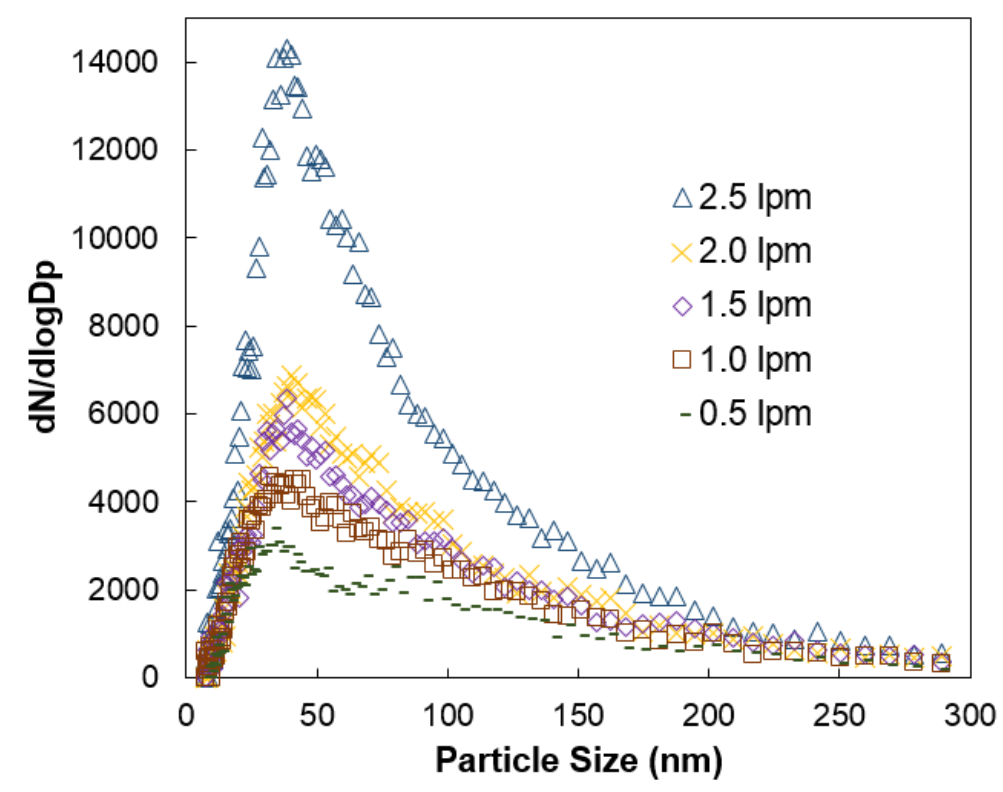

Figure S3: Non-normalized particle size distributions in the bioreactor at different airflow rates. 